\title{
Digital Health Innovations to Improve Cardiovascular Disease Care
}

\author{
Karla Santo $^{1,2,3}$ (D) Julie Redfern ${ }^{2,3}$ \\ Accepted: 10 September 2020 / Published online: 3 October 2020 \\ (C) Springer Science+Business Media, LLC, part of Springer Nature 2020
}

\begin{abstract}
Purpose of Review To review the current evidence supporting the use of digital health technologies in cardiovascular disease (CVD) care.

Recent Findings Studies have evaluated the impact of the use of digital health technologies to improve CVD outcomes through several modalities: text-messaging programmes, smartphone applications (apps) and wearable devices. Text-messaging programmes are to date the most studied type of digital health interventions, and studies have demonstrated reduced CVD risk and improved medication adherence. Literature supporting the use of smartphone apps is also growing but remains limited, with some studies favouring the use of health apps but others showing negative results. Wearable devices are the latest type of technology investigated, and studies have shown positive outcomes in terms of physical activity and detection of arrhythmias. Summary Digital health is a growing and evolving area of investigation. To date, the scientific evidence overall supports the use of such technologies in CVD care and management. Future research using new models are needed to continue to evaluate these new technologies.
\end{abstract}

Keywords Digital Health · eHealth · mHealth · Digital technology · Cardiovascular disease

\section{Introduction}

\section{Cardiovascular Diseases: Burden, Risk Factors and Management}

Cardiovascular diseases (CVD) are the leading cause of death globally. In 2016, the global prevalence of CVD was estimated to be approximately 470 million [1] and there were

This article is part of the Topical Collection on Coronary Heart Disease

Karla Santo

karla.santo@einstein.br

Julie Redfern

julie.redfern@sydney.edu.au

1 Academic Research Organization, Hospital Israelita Albert Einstein, Av. Albert Einstein 627, Bloco A, 2o subsolo, São Paulo, SP CEP 05652-900, Brazil

2 Westmead Applied Research Centre, Westmead Clinical School, Faculty of Medicine and Health, The University of Sydney, Sydney, Australia

3 Cardiovascular Division, The George Institute for Global Health, Sydney, Australia approximately 17.6 million deaths due to CVD worldwide [2]. Furthermore, the number of deaths due to CVD increased about 15\% between 2006 and 2016, and this increase was mainly due to an increase in deaths due to ischaemic heart disease [2]. Importantly, over three quarters of these deaths occurred in low- and middle-income countries, where the cardiovascular mortality rates are substantially higher than those in high-income countries [3].

The term CVD encompasses a wide range of diseases, including ischaemic heart disease, cerebrovascular disease, hypertensive heart disease, peripheral vascular disease, rheumatic heart disease, cardiomyopathies and arrhythmias, among others. The majority of CVD is related to a combination of behavioural and metabolic risk factors. Important modifiable behavioural risk factors include smoking, physical inactivity and unhealthy diet. The long-term presence of behavioural risk factors can lead to the metabolic risk factors, such as hypertension, diabetes, dyslipidaemia and overweight/obesity.

The cornerstone of CVD management and prevention is based on interventions to motivate lifestyle modification and adherence to effective cardiovascular medications. Successful strategies to promote smoking cessation, increase physical activity levels, motivate healthy diet and improve medication adherence are associated with improvements in morbidity and reductions in mortality $[4,5]$. However, given the millions of 
people at risk for or with CVD, there are practical, logistical, geographical and financial challenges associated with delivering comprehensive risk factor management to diverse populations. Health systems throughout the world are charged with finding ways to reach more people in efficient and scalable ways.

\section{Digital Health}

Digital Health is defined as the use of digital, mobile and wireless technologies to support the achievement of health objectives [6]. Recently, the term digital health is most often used to describe the use of information and communications technologies for health, and encompasses eHealth and mHealth interventions, as well as emerging fields such as big data analytics, artificial intelligence (AI) and machine learning $[7,8]$. Digital health technologies are transforming healthcare by facilitating disease prevention, diagnosis and management, which in turn empower patients and healthcare professionals to achieve better health outcomes [9].

In a resolution published in 2018, the World Health Organization (WHO) urged health organizations from around the globe to prioritize the development, evaluation, implementation and expansion of digital health technologies and integrate these new technologies into existing health systems infrastructures $[8,10]$. As stated by the WHO Director-General in another report published in 2019, digital health has the potential to close the gap of health access inequities, by improving reach and strengthening health systems [8]. The potential of digital health interventions has become even more relevant during the current COVID-19 pandemic, in which social isolation has boosted the need for rapid proliferation of digital medicine and brought to light some of the regulatory restrictions that make digital expansion difficult [11].

Digital health is a dynamic, ever-evolving field that requires new approaches to assess the efficacy and effectiveness of these innovative technologies. To address this need, WHO has released guidance on how to monitor and evaluate digital health technologies [6]. In this guide, several recommendations are presented, including how to select the study design and indicators to best evaluate a digital health intervention, key components and tools for digital health monitoring, different approaches on data quality assessment, and guidelines for reporting the findings, particularly the mHealth Evidence Reporting and Assessment (mERA) checklist. [6, 12] The guide also highlights the importance of including quantitative as well as qualitative and economic methods in the evaluation of the digital health interventions. [6] In addition, the United States (US) Food and Drug Administration (FDA) is also reshaping its oversight and regulation over digital health technologies, by recognizing that a more efficient, risk-based approach is necessary to appropriately regulate digital health technologies while continuing to foster innovation [9]. One of FDA's new approaches to digital health oversight was the creation of the Digital Health Program, in which several policies were created to allow lower risk technologies to be readily available to the public while assuring that these digital technologies are high quality, safe and effective. While digital health interventions have the potential for significant, largescale impact on healthcare, the implementation of such interventions must be done with a careful evaluation of the scientific evidence of the risks and benefits of these interventions so that resources are not wasted on ineffective interventions [8].

\section{Digital Health Interventions to Improve CVD Care}

As it pertains to CVD, digital health technologies have many potential applications. For example, for diagnostic purposes, an echocardiogram transducer connected to smartphone or tablet can be useful in the diagnosis of valvular heart disease in limited-resource income settings, where a standard echocardiogram machine is not available. In addition, wearable devices, such as smartwatches, can aid the diagnosis of arrhythmias that require continuous rhythm monitoring, where long periods of monitoring would previously have only be feasible with the implantation of a loop recorder. For monitoring purposes, inflatable blood pressure (BP) cuffs connected to a smartphone app via Bluetooth can facilitate home BP monitoring to guide treatment. For treatment purposes, 3-D printing could be used as a potential solution to produce low-cost cardiac valves for valve replacement in the future. Furthermore, AI can be used to assist the development of new medicines and machine learning to predict CVD outcomes.

Although digital health has a huge potential to improve CVD prevention and care, there is limited robust scientific evidence to date to support the use of digital health technologies. In this section, we will review the evidence on digital health interventions in CVD. We will focus on the three main types of interventions that have been most studied to date and are supported by rigorous well-conducted clinical trials: (i) text-messaging programmes, (ii) smartphone applications (apps) and (iii) wearable devices.

\section{Text-Messaging Programmes}

Text-messaging programmes are the most studied type of digital health interventions, being a relatively old technology from the early 2000s. Early on when they started being used, text messages became a convenient, frequent and inexpensive way of communication that did not require Internet connection. Within the healthcare field, text messages also became an attractive tool for communication with patients, as text messages can be sent to a large number of people at once with minimal human input through an automated software, can be 
bought at a bulk-rate reducing the cost to the provider and is usually perceived as a non-intrusive way of communication. Moreover, interventions delivered via text message can reach people who have limited access to healthcare, such as those living in rural and remote areas. In the context of CVD, text messages have been used to improve two cornerstone aspects of CVD management: lifestyle behaviours and medication adherence.

Smoking was one of the first lifestyle behaviours to be addressed via text-messaging programmes. Several studies aimed to assess whether text messages could improve smoking cessation. A Cochrane systematic review published in 2016 reviewed all the available data published up to April 2015 and found 12 studies with a total of 11,885 patients [13••]. The meta-analysis results showed that participants who received the text-messaging programmes were almost twice as likely (relative risk (RR) 1.67, 95\% confidence interval (CI) 1.461.90) to quit smoking at 6 months compared with participants who did not receive the programme. All studies were of good quality and, therefore, added to the strength of evidence. Given these findings, governments and medical organizations are now offering free text-messaging programmes to support those who wish to quit smoking, such as the SmokefreeTXT programme in the USA [14] and the Short Messages against Tobacco (SMAT) programme in Canada [15].

Physical inactivity is another lifestyle behaviour studied in many text-messaging trials. A systematic review recently published evaluated whether text-messaging programmes were effective in promoting physical activity [16••]. The authors reviewed the published data up to December 2017 and found 57 studies with a total of 8742 patients. However, only 13 randomized controlled trials (RCTs) with a total of $1346 \mathrm{pa}-$ tients used objectively measured physical activity outcomes through pedometers and accelerometers. A meta-analysis of 10 of these RCTs found a significant increase in step counts per day (Cohen's $d=0.38,95 \%$ CI $0.19-0.58$ ) at a median follow-up of 12 weeks. About half of the RCTs included in the meta-analysis were considered to have medium to high risk of bias; however, in a sensitivity analysis excluding these lowquality trials, the results did not change significantly (Cohen's $d=0.37,95 \%$ CI $0.11-0.64$ ).

BP and weight management have also been evaluated in text-messaging studies. A recent 2019 systematic review investigated the effects of text-messaging interventions on $\mathrm{BP}$ levels and body mass index (BMI) [17••]. This included nine RCTs, with a total of 3779 participants. and the meta-analyses results demonstrated a reduction of $4.13 \mathrm{mmHg}(95 \% \mathrm{CI}-$ 11.07 to $-2.81 \mathrm{mmHg}, p<0.0001$ ) in systolic BP, of $1.11 \mathrm{mmHg}(95 \% \mathrm{CI}-1.91$ to $-0.31 \mathrm{mmHg}, p=0.002)$ in diastolic BP and of $0.32 \mathrm{~kg} / \mathrm{m} 2(95 \% \mathrm{CI}-0.49$ to $0.158 \mathrm{mmHg}, p<0.0001)$ in BMI. However, individual patient data meta-analyses of five of these trials with 1976 participants did not confirm the results. Another recent 2020 meta-analysis confirmed the benefits of text-messaging programmes on weight loss in overweight participants [18•].

Medication adherence is the other cornerstone of CVD management, and like lifestyle behaviours, several studies have evaluated text-messaging programmes to improve adherence. A Cochrane systematic review, published in 2017, aimed to assess the effects of text-messaging interventions on medication adherence in CVD patients [19••]. Only seven RCTs with a total of 1310 patients were included in the review. It was not possible to conduct a meta-analysis as there were heterogeneities in the way the adherence outcomes were measured, but the authors reported that six out of the seven trials showed beneficial effects of text-messaging programmes on medication adherence in CVD patients. Although promising, it is important to mention that the included studies were small, and the quality of the evidence was considered to be low. Another 2016 systematic review published performed a meta-analysis of RCTs aimed at improving adherence through text messages [20 ]. Although the included studies were not restricted to CVD patients, and included studies evaluating patients with a variety of chronic conditions, this study reported that text-messaging interventions resulted in an approximate $18 \%$ absolute increase in adherence rates.

Importantly, most of the text-messaging studies have evaluated its impact on a single health behaviour. Although improving a specific health behaviour is beneficial to people living with CVD, improvements in multiple behaviours simultaneously are likely to provide a more significant health impact. The TEXT ME study, published in 2015, was one of the few robust RCTs published to date that investigated the effects of text messages on multiple health behaviours [21, 22]. Briefly, TEXT ME was a 6-month text-messaging programme based on behavioural psychology that focused on motivating and supporting change in multiple lifestyle behaviours in patients with coronary heart disease. [23] The study included 710 patients and found that the text-messaging programme was associated with lower total and LDL cholesterol, lower BP, lower BMI, lower smoking rates, higher physical activity levels and higher adherence to dietary guidelines [22, 24, 25]. Of note, participants in the text-messaging group were almost three times more likely to achieve multiple risk factor control than the usual care group (RR 2.80, 95\% CI 1.95-4.02, for achieving 4 or more key CVD risk factors). Other very important findings of this study were that the text-messaging intervention was engaging and useful for patients, was predicted to lead to better clinical outcomes and was cost-saving in a modelling analysis $[26,27]$.

\section{Smartphone Apps}

With the advent of smartphones and tablets, digital health interventions delivered via these new devices started to grow exponentially. Interventions delivered via smartphones and 
tablets are more sophisticated than text-message interventions, as these newer devices are capable of running apps, which are computer-like programmes with many functionalities. Apps can educate patients through written and visual information and monitor and manage health conditions through in-built diaries and automatic reminders. Although there is a huge potential for health apps, there is one important aspect to consider. Different from text-messaging programmes, health apps are often developed with no or little input from health professionals and are not guided by evidence-based guidelines [28]. Hundreds of thousands of health apps are made available to the general public in the app stores with little investigation into whether such apps are beneficial or harmful to patients.

Given the many potential functionalities of a health app, it is important to build an app that focuses on features most likely to impact an individual's behaviour. An interesting review of the evolution of smartphone apps in preventing CVD was published in 2015 [29•]. The authors discuss that CVD prevention apps should have some core components to promote behaviour change. They conclude these apps should be simple, have credible information, be based on behavioural change concepts, use real-time data tracking for monitoring behaviours, provide rewards to motivate patients to keep on track, have personalized information, have social elements to engage patients and ensure privacy.

A recent systematic review published in 2018 aimed to evaluate the effectiveness, acceptability and usefulness of health apps for CVD management and control [30••]. The authors reviewed the literature published up to April 2017 and found 10 studies with a total of 607 patients. The interventions were varied and targeted different populations of patients with hypertension, coronary heart disease, heart failure and stroke. Only three studies were RCTs, and due to the heterogeneity of the studies, a meta-analysis was not carried out. The results showed that app users had improvements in BP, BMI, waist circumference, cholesterol, physical activity, smoking cessation and medication adherence, as well as quality of life, disease-specific knowledge, psychological wellbeing and re-hospitalization rates. This review also evaluated app characteristics and found that appealing app features included tracking of health behaviours, self-monitoring, disease information and personalized/customisable content. Although the results point to a potential benefit of health apps for CVD care, the authors caution that low-quality of evidence to date, given the small number of RCTs, small sample sizes and short duration of the interventions, limit interpretation of these results.

Since the publication of the afore-mentioned review, three relevant RCTs have been published. The results of these RCTs are presented in Table 1. The study by Morawski et al. was published in 2018 and aimed to assess whether a smartphone app improved medication adherence and BP control in patients with uncontrolled hypertension [31, 35].
An interesting aspect of this trial was that it was an entirely virtual RCT with no in-person visits. Recruitment was done via social media, online patient communities and advertisements; informed consent was provided via the study website; eligibility assessment was conducted via an online questionnaire; and home BP measurements performed using a Bluetooth-enabled automated BP monitor mailed by the study team. At 12 weeks, the results showed an improvement in self-reported medication adherence and no significant difference in systolic BP in the app users compared with nonapp users.

A second trial published by Santo et al. in 2019 also aimed to assess whether medication reminder apps improved medication adherence and other clinical outcomes, such as BP and cholesterol levels $[32,36]$. This study was a 3-arm RCT comparing a basic medication reminder app, an advanced reminder app and usual care (see Table 1 for intervention details) in a coronary heart disease population. Similar to the previous trial, the results showed an improvement in self-reported medication adherence that was not accompanied by any statistically significant improvements in clinical outcomes in app users compared with usual care. Of note, a mixed-methods evaluation of this trial found that the majority of patients who received the app intervention found useful to have an easily accessible record of their medications names and dosages on their smartphones and that the medication reminders helped them take their medications correctly [37]. In terms of usability and engagement, most patients found the app was easy-touse and used the medication reminders at least once a day, while only a small number of patients have technical difficulties while using the app.

A few interesting aspects of both of these trials should be discussed. Both studies had a small but statistically significant improvement in self-reported medication adherence at a shortterm follow-up of 3 months. First, the magnitude of effect might have been too small, and second, the follow-up period might not have been long enough to translate the benefits of improved medication adherence into clinical outcomes, such as BP and cholesterol levels. Of note, both trials reported that some participants in the intervention groups did not download the apps and, therefore, did not receive the intervention, while some participants in the control group downloaded a medication reminder app during the course of the trial. Both of these situations could also have contributed to the null effect in the clinical outcomes.

The third relevant trial by Redfern et al. assessed whether a consumer-directed digital health strategy for CVD risk management, which included a website and an accompanying app (CONNECT), would improve medication adherence and clinical outcomes in patients with moderate to high risk of CVD [33, 34•, 38]. The intervention was integrated with the patient's electronic health record at the primary healthcare facility. At 12 months, the study results did not show a statistically 
Table 1 Recent randomized clinical trials of app-based interventions in CVD patients

\begin{tabular}{|c|c|c|c|c|c|c|}
\hline $\begin{array}{l}\text { Author, } \\
\text { year } \\
\text { Country }\end{array}$ & $\begin{array}{l}\text { Number of } \\
\text { participants }\end{array}$ & Duration & $\begin{array}{l}\text { Population } \\
\text { characteristics }\end{array}$ & Interventions & Outcomes & $\begin{array}{l}p \\
\text { value }\end{array}$ \\
\hline $\begin{array}{c}\text { Morawski, } \\
{[31 \bullet]} \\
2018 \\
\text { USA }\end{array}$ & 412 & 12 weeks & $\begin{array}{l}\text { Mean age: } \\
52 \text { years } \\
\text { (SD: NP) } \\
\text { Males: } 165 \\
(40 \%)\end{array}$ & $\begin{array}{l}\text { Intervention: Medisafe app } \\
\text { - BP monitoring/tracking } \\
\text { - Medication list } \\
\text { - Medication reminders } \\
\text { - Medication adherence weekly reports } \\
\text { - Medfriend feature, in which the user would allow app access to a } \\
\text { family member or friend to view the medication-taking history, } \\
\text { receive alerts when the user would miss a medication dose and } \\
\text { provide peer-support } \\
\text { Control: Usual care }\end{array}$ & $\begin{array}{l}\text { Co-primary outcome: } \\
\text { SBP-mean }(\mathrm{SD}) \\
\text { I: } 140.8 \mathrm{mmHg}(15.7) \\
\text { C: } 141.2 \mathrm{mmHg}(17.3) \\
\text { Co-primary outcome: } \\
\text { Medication adherence } \\
\quad \text { MMAS-8 score-mean } \\
\quad \text { (SD) } \\
\text { I: } 6.3(1.6) \\
\text { C: } 5.7(1.8) \\
\text { Secondary outcome: } \\
\text { SBP/DBP < } 140 / 90 \mathrm{mmHg}- \\
\quad n(\%) \\
\text { I: } 67(35.8) \\
\text { C: } 69(37.9)\end{array}$ & 0.78 \\
\hline $\begin{array}{c}\text { Santo, } \\
{[32 \cdot]} \\
2019 \\
\text { Australia }\end{array}$ & 163 & 3 months & $\begin{array}{c}\text { Mean age: } \\
57.9 \text { years } \\
\text { (SD 8.91) } \\
\text { Males: } 143 \\
(87.7 \%)\end{array}$ & $\begin{array}{l}\text { Intervention 1: Basic app } \\
\text { - Simple non-interactive daily reminders (one-time only), similar } \\
\text { to an alarm or text message, for medication-taking at the correct } \\
\text { time every day } \\
\text { Intervention 2: Advanced app } \\
\text { - Interactive and customizable features } \\
\text { - Daily reminders that could be snoozed, rescheduled or marked as } \\
\text { 'taken' or 'missed' } \\
\text { - Default settings for the reminders to occur up to } 3 \text { times at } \\
\text { 10-min intervals } \\
\text { - Medication refill reminders } \\
\text { - Medication adherence statistics } \\
\text { - Ability to export and share information with others } \\
\text { - Ability to alert other people if the participant missed a } \\
\text { medication dose } \\
\text { Control: Usual care }\end{array}$ & $\begin{array}{l}\text { Primary outcome: } \\
\text { Medication adherence } \\
\quad \text { MMAS-8 score-mean } \\
\quad(95 \% \text { CI }) \\
\text { I: } 7.11(6.90-7.31) \\
\text { C: } 6.63(6.35-6.92) \\
\text { Secondary outcome: } \\
\text { SBP-mean (SD) } \\
\text { I: } 125.0 \text { (17.05) } \\
\text { C: } 126.0(19.12) \\
\text { Secondary outcome: } \\
\text { DBP-mean (SD) } \\
\text { I: } 80.2(8.76) \\
\text { C: } 81.1 \text { (10.47) } \\
\text { Secondary outcome: } \\
\text { Total cholesterol—-mean (SD) } \\
\text { I: } 3.60 \text { ( } 0.90) \\
\text { C: } 3.60 \text { ( } 0.84) \\
\text { Secondary outcome: } \\
\text { LDL cholesterol—-mean (SD) } \\
\text { I: } 1.62(0.75) \\
\text { C: } 1.68(0.70)\end{array}$ & 0.592 \\
\hline $\begin{array}{l}\text { Redfern, } \\
{[33,34 \cdot]} \\
2019\end{array}$ & 934 & 12 months & $\begin{array}{l}\text { Mean age: } \\
67.0 \text { years } \\
(\mathrm{SD} 8.1)\end{array}$ & $\begin{array}{l}\text { Intervention: CONNECT website/app } \\
\text { - Integration with patient's electronic health record with the ability } \\
\text { to view personal health information, such as prescribed }\end{array}$ & $\begin{array}{l}\text { Medication adherence_- } \% * \\
\text { I: } 32.8 \\
\text { C: } 29.9\end{array}$ & 0.485 \\
\hline Australia & & & $\begin{array}{c}\text { Males: } 719 \\
(77 \%)\end{array}$ & $\begin{array}{l}\text { medicines, test results, BP and weight } \\
\text { - Heart age calculator } \\
\text { - Simple medication and lifestyle reminders } \\
\text { - Appointment reminders } \\
\text { - Motivational message prompts, e.g. salt reduction tips } \\
\text { - Interactive goal setting } \\
\text { - Peer forums } \\
\text { Control: Usual care }\end{array}$ & $\begin{array}{l}\text { SBP-mean (SD) } \\
\text { I: } 136.4(17.6) \\
\text { C: } 136.3(16.1) \\
\text { LDL cholesterol—mean (SD) } \\
\text { I: } 2.40(1.00) \\
\text { C: } 2.50(0.90) \\
\text { BMI-mean (SD) } \\
\text { I: } 29.7(5.70) \\
\text { C: } 29.4(5.00) \\
\text { Physically active—\% } \\
\text { I: } 87 \\
\text { C: } 79.7 \\
\text { Current smoker-\% } \\
\text { I: } 8.1 \\
\text { C: } 12 \\
\text { eHealth literacy (eHEALS } \\
\text { score)-mean (SD) } \\
\text { I: } 28.3 \text { (6.3) } \\
\text { C: } 26.4(7.5)\end{array}$ & 0.921 \\
\hline
\end{tabular}

BMI, body mass index; C, control group; CI, confidence interval; DBP, diastolic blood pressure; I, intervention group; LDL, low-density lipoprotein; n, number; NP, not provided; SBP, systolic blood pressure; SD, standard deviation

*Medication adherence defined as taking BP and cholesterol medications on $\geq 80 \%$ of days 
significant improvement in medication adherence in the intervention group compared with the control group. However, there were more intervention participants who were physically active and had high eHealth literacy than control participants. Interestingly, qualitative research of participants in the CONNECT trial found that $87 \%$ of primary care physicians said they would recommend the strategy. Also, as a result of the CONENCT intervention, $31 \%$ of patients reported improved medication adherence, $40 \%$ reported doing more to improve mental health and well-being, $47 \%$ reported higher weekly physical activity and $61 \%$ reported healthier eating habits [39]. Taken together, the results of the CONNECT trial highlight potential health value usefulness for patients despite no significant improvement in medication adherence.

Taken together, the three recent RCTs described above provide insight into the assessment of outcome measures and the potential value for patients, and the importance of this when evaluating digital health interventions. The trials highlight the importance of patient engagement and interest in the intervention and the alignment of those aspects with perceived benefit. As stated in the WHO guide on monitoring and evaluating digital health interventions, it is essential to monitor the digital intervention in terms of whether the intervention worked as it was intended to, as well as how much the user interacted with the technology [6]. Therefore, it is extremely important to engage different stakeholders (including health professionals and consumers) in codesigning the digital health interventions to maximize the potential benefit of such interventions. Another aspect is engaging key players in the delivery of the intervention; for example, if the apps are prescribed by a physician, patients might feel more motivated to engage with the app than downloading an app on their own or even as part of a research study. In the aforementioned RCTs, there was no physician involvement in reinforcing the use of the apps, and thus, this might have contributed to some of the trial results. Finally, the RCTs highlight challenges with assessing outcomes that are clinically measurable but also meaningful to patients. It is important that digital health interventions be developed and evaluated in collaboration with end users and that patient-reported outcomes and experience measures are considered in the final evaluation.

\section{Wearable Devices}

Wearable devices are electronic devices that can be worn and have the capability to capture information, perform data processing and provide output of relevant information through a connection with another device, such as a smartphone app, or by itself [40]. Given these characteristics, wearable devices have become increasingly popular in healthcare settings as they can be used as real-time tools to continuously monitor data such as vital signs, activities and behaviours. This relevant information provided by wearable devices can, in turn, assist health professionals in making informed clinical decisions.

In the context of CVD care and management, the first major lifestyle factor that wearable devices targeted was physical activity. Consumer-based activity trackers, such as Fitbit, are now widely available in stores around the world with accessible prices. These activity trackers provide information on daily step counts, distance walked, energy expenditure and heart rate. When connected to smartphone apps, activity trackers can be motivational tools, reminding individuals to be physically active, providing virtual rewards when a goal is achieved (e.g. the user gets 'stars' when achieving 10,000 steps in a day) and offering peer support by sharing performance results to stimulate a competition-like environment.

Given the wide availability and increasing popularity of these consumer-based activity trackers, many studies have investigated whether the use of such gadgets can improve physical activity levels. A recent systematic review published in 2019 aimed to investigate the effects of interventions that used consumer-based activity trackers compared with no activity tracker use on physical activity participation and sedentary behaviour [41 • $]$. The authors reviewed studies published up to March 2017 and included 28 RCTs in the review with a total of 3646 participants. Of these, 11 trials provided objective outcome data on the number of steps per day and were pooled in a meta-analysis that showed a significant increase in steps per day, an increase of approximately 627 steps per day (95\% CI 417-862 steps per day), in the arm that used activity trackers compared with the one that did not (standardized mean difference (SMD) 0.23, 95\% CI 0.15-0.32, $p<0.001$ ). In addition, 12 trials measured moderate-to-vigorous physical activity (MVPA) levels and were also pooled in a metaanalysis that showed a significant increase in minutes per day spent in MVPA, approximately 75 min per day increase (95\% CI 42-109 min per day) in the activity tracker users compared with non-users (SMD 0.28, 95\% CI 0.14-0.41, $p<0.001)$. However, no significant difference in sedentary behaviour was found. An interesting aspect found in this review was that interventions that were classified as multi-faceted, in which the consumer-based activity trackers were only one component of the intervention, had a greater impact on physical activity levels compared with interventions in which the wearable devices were the only component of the intervention.

Specifically, in a population of patients with CVD, another systematic review also aimed to evaluate the use of the same type of wearable devices on physical activity outcomes [42••]. This review reviewed studies from January 2000 to May 2018 and found 35 eligible studies with a total of 4528 patients. Of these, 28 trials were included in the meta-analyses. For the steps per day outcome, the meta-analysis results of 17 RCTs showed a statistically significant increase with a mean 
Fig. 1 Apple Heart Study results [44"*]

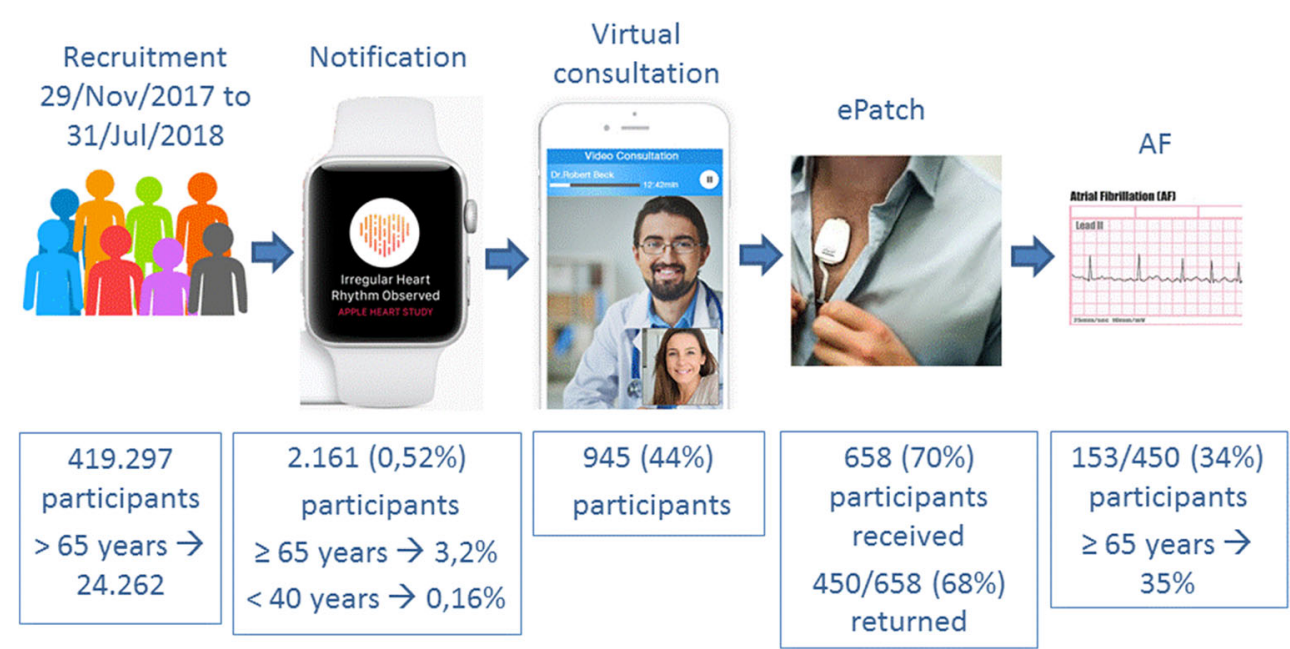

difference of 2592.33 steps per day (95\% CI 1688.62 3496.04 steps per day, $p<0.00001$ ) in favour of the use of wearable devices. In terms of weekly minutes of MVPA, the meta-analysis results of 12 RCTs showed a statistically significant increase with a mean difference of 36.31 min per week (95\% CI 18.33-54.29 min per week, $p<0.0001$ ) in favour of the intervention.

Wearable devices have the potential to impact other CVDrelated outcomes, such as weight, BP, diabetes and cholesterol. To investigate the effects of wearable devices on these outcomes, a systematic review was conducted in 2019, reviewing the available data up to October 2018 [43•]. This review found only 6 studies with 1615 participants. In regard to effects on weight management, five trials provided weight loss outcomes; however, only one showed a significant weight reduction in 47 adults with $\mathrm{BMI} \geq 23 \mathrm{~kg} / \mathrm{m}^{2}$ and sleep apnoea. Regarding BP control, three studies evaluated BP outcomes with two of them reporting improved systolic BP with the intervention. In terms of cholesterol management, two studies reported outcomes on total, LDL and HDL cholesterol and presented conflicting results, where in one study there were significant improvements in total cholesterol and in the other study a significant improvement is seen only in HDL cholesterol. Diabetes management was also assessed in one study and demonstrated a significant reduction in $\mathrm{HbAlc}$.

More recently, a more sophisticated type of wearable devices, the smartwatches, have become a potential tool to monitor heart rhythm and detect arrhythmias. The Apple Heart Study was a ground-breaking study, published in 2019, that aimed to evaluate the ability of an irregular pulse notification algorithm to detect atrial fibrillation (AF) with the use a smartwatch, the Apple Watch [44••]. The study was an entirely virtual pragmatic study, in which potential participants were any individual who owned an Apple Watch and a compatible iPhone, were 22 years or older, resided in the USA, spoke English, did not have a previous history of AF and were not currently taking anti-coagulants. Potential participants had to download the study app in the app store to check eligibility and provide informed consent before the irregular pulse notification was activated on the smartwatch. Upon activation, the app algorithm would evaluate the heart rhythm using the smartwatch photoplethysmography sensor, which uses LED lights to intermittently measure changes in blood flow. These pulse signals were used to generate a pulse interval graph, called a tachogram, which was then classified as regular or irregular. A participant would receive an irregular pulse notification if 5 out of 6 tachograms were considered irregular in $48 \mathrm{~h}$. If the participant received this irregular pulse notification, he/she was scheduled for a telemedicine consultation with a physician who would evaluate whether the case required further evaluation. If so, participants were mailed an ECG patch to wear for up to 7 days to confirm the arrhythmia. The study results are presented in Fig. 1. Based on the results presented in Fig. 1, the authors report that the positive predictive value of a single irregular tachogram was $0.71(95 \% \mathrm{CI}$ $0.69-0.74)$, meaning that $71 \%$ of the time when a tachogram detected an irregular pulse, AF was also detected by the ECG patch. Meanwhile, the positive predictive value of the irregular pulse notification was 0.84 (95\% CI 0.76-0.92), meaning that $84 \%$ of the times when a participant received an irregular pulse notification, the participant had confirmed AF on the simultaneous ECG. There are a few key points to discuss about this study. First, the huge number of recruited patients in a short period of 8 months is impressive, demonstrating that new models of virtual studies where data is collected directly from the participant, without the direct involvement of a research team, may be the way forward for clinical studies. Second, wearable devices, such as smartwatches, that are now already being used by millions of consumers can be used to provide meaningful information, through big data analyses, that can provide prompts to motivate people to seek medical care for further evaluation of a significant medical condition. Third, this study also opens up new opportunities for collaborations between technology companies, which are 
manufacturing these innovative gadgets and already collecting data from consumers, and researchers; and these new collaborations can provide new insights into improving healthcare of patients.

\section{Conclusion}

Digital health is a growing and evolving area of investigation. To date, text-messaging programmes are the type of digital health interventions that are most supported by scientific evidence with studies showing potential benefits for smoking cessation, physical activity, blood pressure and cholesterol control, weight management and medication adherence. The literature to support the use of smartphone apps in CVD prevention is growing, but it is still limited. Small studies have favoured the use of health apps for improved CVD health outcomes; however, recent RCTs have not confirmed benefits associated with the use of health apps. Wearable devices are the latest type of technology investigated in clinical studies. Studies support the use of physical activity trackers on improving physical activity levels, while smartwatches can be used as a novel tool to detect arrhythmias. Importantly, digital technology does have the potential to overcome several barriers, such as geography and time, and hence, even with equivalent health outcomes to standard care, there are enormous positives in terms of reach from a public health perspective. Furthermore, digital health interventions should not be viewed as a stand-alone, one-sizefits-all solution but rather an addition to the current multifaceted health interventions that can be personalized to different types of patient populations. With the digital health revolution, we can not only consider these tools as a way to achieve better patient health outcomes but also as innovative ways to reimagine the way we currently conduct clinical studies. These digital technologies can enable new models of virtual trials, potentially reducing the duration and costs of future research, while increasing our ability to achieving meaningful results.

Acknowledgements JR is funded by a National Health and Medical Research Council Career Development Fellowship (APP1143538).

Authors' Contributions KS drafted the manuscript. KS and JR have read and approved the final manuscript.

\section{Compliance with Ethical Standards}

Conflict of Interest Karla Santo and Julie Redfern declare no conflicts of interest relevant to this manuscript.

Human and Animal Rights and Informed Consent This article does not contain any studies with human or animal subjects performed by any of the authors.

\section{References}

Papers of particular interest, published recently, have been highlighted as:

- Of importance

- Of major importance

1. Abajobir AA, Abate KH, Abbafati C, Abbas KM, Abd-Allah F, Abdulkader RS, et al. Global, regional, and national incidence, prevalence, and years lived with disability for 328 diseases and injuries for 195 countries, 1990-2016: a systematic analysis for the global burden of disease study 2016 . Lancet. 2017;390(10100):1211-59.

2. Abajobir AA, Abbafati C, Abbas KM, Abd-Allah F, Abera SF, Aboyans V, et al. Global, regional, and national age-sex specific mortality for 264 causes of death, 1980-2016: a systematic analysis for the global burden of disease study 2016. Lancet. 2017;390(10100):1151-210.

3. World health statistics 2017: monitoring health for the SDGs, Sustainable Development Goals. Geneva: World Health Organization, 2017.

4. Chow CK, Jolly S, Rao-Melacini P, Fox KAA, Anand SS, Yusuf S. Association of diet, exercise, and smoking modification with risk of early cardiovascular events after acute coronary syndromes. Circulation. 2010;121(6):750-8.

5. Artinian NT, Fletcher GF, Mozaffarian D, Kris-Etherton P, van Horn L, Lichtenstein AH, et al. Interventions to promote physical activity and dietary lifestyle changes for cardiovascular risk factor reduction in adults: a scientific statement from the American Heart Association. Circulation. 2010;122(4):406-41.

6. Monitoring and evaluating digital health interventions: a practical guide to conducting research and assessment. Geneva: World Health Organization, 2016.

7. Digital Health. US Food and Drug Administration website. [Accessed on 27 May 2020]. Available from: https://www.fda. gov/medical-devices/digital-health.

8. WHO guideline: recommendations on digital interventions for health system strengthening. Geneva: World Health Organization, 2019.

9. Digital Health Innovation Action Plan. US Food and Drug Administration, 2018.

10. Digital Health Resolution. Geneva: World Health Organization, 2018.

11. Keesara S, Jonas A, Schulman K. Covid-19 and health care's digital revolution. N Engl J Med. 2020;382(23):e82.

12. Agarwal S, LeFevre AE, Lee J, et al. Guidelines for reporting of health interventions using mobile phones: mobile health (mHealth) evidence reporting and assessment (mERA) checklist. BMJ. 2016;352:11174.

13.• Whittaker R, McRobbie H, Bullen C, Rodgers A, Gu Y. Mobile phone-based interventions for smoking cessation. Cochrane Database Syst Rev. 2016;4 Recent Cochrane systematic review with meta-analysis demonstrating benefits of text-messaging programmes for smoking cessation.

14. SmokefreeTXT United States [cited 201716 December]. Available from: https://smokefree.gov/smokefreetxt.

15. Short Messages Against Tobbaco (SMAT) Canada [cited 201716 December]. Available from: https://www.smat.ca/en.

16.• Smith DM, Duque L, Huffman JC, Healy BC, Celano CM. Text Message Interventions for Physical Activity: A Systematic Review and Meta-Analysis. Am J Prev Med. 2020;58(1):14251 Recent systematic review with meta-analysis demonstrating that text-messaging interventions are associated with improved physical activity. 
17.• Shariful Islam SM, Farmer AJ, Bobrow K, Maddison R, Whittaker R, Pfaeffli Dale LA, et al. Mobile phone textmessaging interventions aimed to prevent cardiovascular diseases (Text2PreventCVD): systematic review and individual patient data meta-analysis. Open Heart. 2019;6(2):e001017 Recent systematic review with meta-analysis demonstrating improvements on systolic blood pressure and body mass index with text-messaging interventions.

18. Skinner R, Gonet V, Currie S, Hoddinott P, Dombrowski SU. A systematic review with meta-analyses of text message-delivered behaviour change interventions for weight loss and weight loss maintenance. Obes Rev. 2020;21(6):e12999 Recent systematic review with meta-analysis demonstrating the benefits of textmessaging programmes on weight loss.

19.• Adler AJ, Martin N, Mariani J, Tajer CD, Owolabi OO, Free C, et al. Mobile phone text messaging to improve medication adherence in secondary prevention of cardiovascular disease. Cochrane Database Syst Rev. 2017;4 Recent systematic review demonstrating the beneficial effects of text-messaging programmes on medication adherence in patients with cardiovascular diseases.

20. Thakkar J, Kurup R, Laba TL, Santo K, Thiagalingam A, Rodgers A, et al. Mobile telephone text messaging for medication adherence in chronic disease: a meta-analysis. JAMA Intern Med. 2016;176(3):340-9 Recent systematic review with metaanalysis demonstrating the beneficial effects of textmessaging programmes on medication adherence in patients with chronic diseases.

21. Chow CK, Redfern J, Thiagalingam A, Jan S, Whittaker R, Hackett $\mathrm{M}$, et al. Design and rationale of the tobacco, exercise and diet messages (TEXT ME) trial of a text message-based intervention for ongoing prevention of cardiovascular disease in people with coronary disease: a randomised controlled trial protocol. BMJ Open. 2012;2(1):e000606.

22. Chow CK, Redfern J, Hillis GS, Thakkar J, Santo K, Hackett ML, et al. Effect of lifestyle-focused text messaging on risk factor modification in patients with coronary heart disease: a randomized clinical trial. Jama. 2015;314(12):1255-63 Randomised clinical trial demonstrating the beneficial effects of a lifestyle-focused textmessaging programme on multiple cardiovascular risk factors.

23. Redfern J, Thiagalingam A, Jan S, Whittaker R, Hackett ML, Mooney J, et al. Development of a set of mobile phone text messages designed for prevention of recurrent cardiovascular events. Eur J Prev Cardiol. 2014;21(4):492-9.

24. Thakkar J, Redfern J, Thiagalingam A, Chow CK. Patterns, predictors and effects of texting intervention on physical activity in CHD insights from the TEXT ME randomized clinical trial. Eur J Prev Cardiol. 2016;23(17):1894-902.

25. Santo K, Hyun K, de Keizer L, Thiagalingam A, Hillis GS, Chalmers J, et al. The effects of a lifestyle-focused text-messaging intervention on adherence to dietary guideline recommendations in patients with coronary heart disease: an analysis of the TEXT ME study. Int J Behav Nutr Phys Act. 2018;15(1):45.

26. Burn E, Nghiem S, Jan S, Redfern J, Rodgers A, Thiagalingam A, et al. Cost-effectiveness of a text message programme for the prevention of recurrent cardiovascular events. Heart. 2017;103(12):893-4.

27. Redfern J, Santo K, Coorey G, Thakkar J, Hackett M, Thiagalingam A, et al. Factors influencing engagement, perceived usefulness and behavioral mechanisms associated with a text message support program. PLoS One. 2016;11(10):e0163929.

28. Santo K, Richtering SS, Chalmers J, Thiagalingam A, Chow KC, Redfern J. Mobile phone apps to improve medication adherence: a systematic stepwise process to identify high-quality apps. JMIR mHealth uHealth. 2016;4(4):e132.
29. Neubeck L, Lowres N, Benjamin EJ, Freedman SB, Coorey G, Redfern J. The mobile revolution-using smartphone apps to prevent cardiovascular disease. Nat Rev Cardiol. 2015;12(6):350-60 An interesting review on the role of smartphone apps in revolutionizing the prevention of cardiovascular diseases.

30.• Coorey GM, Neubeck L, Mulley J, Redfern J. Effectiveness, acceptability and usefulness of mobile applications for cardiovascular disease self-management: systematic review with meta-synthesis of quantitative and qualitative data. Eur J Prev Cardiol. 2018;25(5): 505-21 Recent systematic review demonstrating the beneficial effects of smartphone apps for the management and control of cardiovascular risk factors.

31. Morawski K, Ghazinouri R, Krumme A, Lauffenburger JC, Lu Z, Durfee E, et al. Association of a Smartphone Application with Medication Adherence and Blood Pressure Control: the MedISAFE-BP randomized clinical TrialSmartphone app and blood pressure medication AdherenceSmartphone app and blood pressure medication adherence. JAMA Intern Med. 2018;178(6): 802-9 Randomised clinical trial demonstrating improvements on medication adherence with the use of a smartphone app in hypertensive patients.

32. Santo K, Singleton A, Rogers K, Thiagalingam A, Chalmers J, Chow CK, et al. Medication reminder applications to improve adherence in coronary heart disease: a randomised clinical trial. Heart. 2019;105(4):323 Randomised clinical trial demonstrating improvements on medication adherence with the use of smartphone apps in patients with coronary heart disease.

33. Redfern J, Usherwood T, Coorey G, Mulley J, Scaria A, Neubeck $\mathrm{L}$, et al. P5307A consumer-direct digital health intervention for cardiovascular risk management in primary care: the Consumer Navigation of Electronic Cardiovascular Tools (CONNECT) randomised controlled trial. Eur Heart J. 2019;40(Supplement 1).

34. Redfern J, Coorey G, Mulley J, Scaria A, Pitt C, Weir K, et al. Effectiveness of an integrated responsive web application for cardiovascular disease management in primary care: 1 year multicenter, open-label randomized controlled trial. npg Digit Med. 2020; (In press, accepted $29^{\text {th }}$ July 2020). Randomised clinical trial of a smartphone app and web portal integrated with the patient's electronic health record for cardiovascular risk management.

35. Morawski K, Ghazinouri R, Krumme A, McDonough J, Durfee E, Oley $\mathrm{L}$, et al. Rationale and design of the medication adherence improvement support app for engagement-blood pressure (MedISAFE-BP) trial. Am Heart J. 2017;186:40-7.

36. Santo K, Chow CK, Thiagalingam A, Rogers K, Chalmers J, Redfern J. MEDication reminder APPs to improve medication adherence in coronary heart disease (MedApp-CHD) study: a randomised controlled trial protocol. BMJ Open. 2017;7(10).

37. Santo K, Singleton A, Chow CK, et al. Evaluating reach, acceptability, utility, and engagement with an app-based intervention to improve medication adherence in patients with coronary heart disease in the MedApp-CHD study: a mixed-methods evaluation. Med Sci (Basel, Switzerland). 2019;7(6).

38. Redfern J, Usherwood T, Harris MF, Rodgers A, Hayman N, Panaretto K, et al. A randomised controlled trial of a consumerfocused e-health strategy for cardiovascular risk management in primary care: the consumer navigation of electronic cardiovascular tools (CONNECT) study protocol. BMJ Open. 2014;4(2):e004523.

39. Coorey GM, Peiris D, Usherwood T, Neubeck L, Mulley J, Redfern J. Persuasive design features within a consumer-focused eHealth intervention integrated with the electronic health record: a mixed methods study of effectiveness and acceptability. PLoS One. 2019;14(6):e0218447.

40. Xue Y. A review on intelligent wearables: uses and risks. Human Behav Emerg Technol. 2019;1(4):287-94.

41.• Brickwood K-J, Watson G, O'Brien J, Williams AD. Consumerbased wearable activity trackers increase physical activity 
participation: systematic review and meta-analysis. JMIR mHealth uHealth. 2019;7(4):e11819 Recent systematic review with metaanalysis demonstrating the benefits of using wearable devices on physical activity.

42.• Kirk MA, Amiri M, Pirbaglou M, Ritvo P. Wearable technology and physical activity behavior change in adults with chronic Cardiometabolic disease: a systematic review and meta-analysis. Am J Health Promot. 2019;33(5):778-91 Recent systematic review with meta-analysis demonstrating the benefits of using wearable devices on physical activity in patients with cardiovascular diseases.

43. Jo A, Coronel BD, Coakes CE, Mainous AG. Is there a benefit to patients using wearable devices such as Fitbit or health apps on mobiles? A systematic review. Am J Med. 2019;132(12):1394400.e1 Recent systematic review demonstrating the benefits of using wearable devices on cardiovascular clinical outcomes.

44.• Perez MV, Mahaffey KW, Hedlin H, Rumsfeld JS, Garcia A, Ferris $\mathrm{T}$, et al. Large-scale assessment of a smartwatch to identify atrial fibrillation. N Engl J Med. 2019;381(20):1909-17 First largescale virtual clinical study on the use of a smartwatch to detect atrial fibrillation.

Publisher's Note Springer Nature remains neutral with regard to jurisdictional claims in published maps and institutional affiliations. 